\title{
THE LONG SIDE OF THE MARKET AND THE SHORT END \\ OF THE STICK: BARGAINING POWER AND PRICE \\ FORMATION IN BUYERS', SELLERS', AND \\ BALANCED MARKETS*
}

\author{
CurTis R. TAYLOR
}

The determinants of bargaining power and price formation in a dynamic exchange market where new traders enter randomly over time are studied. When agents on the long side of the market possess the option to wait for the arrival of future partners, the terms of trade in the spot market must honor the value of this option. The equilibrium terms of trade are expressed in intuitive closed-form equations that highlight the distinct influences of short-run spot-market conditions and long-run market demographics.

\section{INTRODUCTION}

Few concepts in economics are as intuitive as the idea that traders on the long side of a market wind up holding the short end of the stick. That is, they receive little or none of the gains from trade. Consider, for instance, the classic setting in which $b$ identical buyers each with unit demands for an indivisible homogeneous good face $s$ identical sellers each with one unit of the good for sale. It is well-known that in a broad class of one-shot trading games, all of the surplus in this setting goes to the short side of the market in equilibrium. That is, if $b<s$, sellers bid the price down to their reservation value in equilibrium, and if $b>s$, buyers bid the price up to their reservation value. Often, it is only when $b=s$ that market institutions (the rules of the game) have any bearing on the equilibrium division of surplus.

This classic result is somewhat brittle because of the discontinuity in the terms of trade when moving from positive to negative levels of excess demand. Its logic is familiar, however, from Bertrand competition, and it is very compelling. The option value to an agent frozen out of a trade in a one-shot market game is his reservation payoff of zero. Any outcome that confers nonzero surplus to a long-side agent cannot be sustained in equilibrium, because all of the long-side agents who would otherwise be frozen out possess a strict incentive to undercut.

*I thank Kenneth Binmore, David Pearce, Larry Samuelson, Lars Stole, Daniel Vincent, and Steven Wiggins. I am especially grateful to Donald Deere, Canice Prendergast, and Samuel Kortum. Financial support from the National Science Foundation (PBR 9422830) is gratefully acknowledged.

(1) 1995 by the President and Fellows of Harvard College and the Massachusetts Institute of Technology.

The Quarterly Journal of Economics, August 1995 
This article extends the classic one-shot trading game to an infinite horizon environment with random arrivals of new traders. Not only does this generalization lend realism to the setting, but also it softens the brittle "all-or-nothing" equilibrium outcome in an intuitive and appealing way. Specifically, the option value from being frozen out in a given period is no longer zero because of the potential arrival of future trading partners. As the level of excess demand increases in absolute value, however, the option value from being frozen out diminishes. The equilibrium terms of trade, therefore, depend monotonically on the level of excess demand, and agents on the short side of the market receive all of the surplus only in the limit.

An important feature of the model is that the equilibrium terms of trade are expressed in intuitive closed-form equations. These solutions highlight the distinct influences of short-run spot-market conditions and long-run market demographics. Buyers' and sellers' markets are defined, respectively, as states in which excess demand in the spot market is negative and positive. These states may persist indefinitely or may eventually be neutralized by the arrival of short-side traders. When agents discount heavily, the degree of imbalance in the spot market plays the central role in determining the equilibrium terms of trade; and when they are patient, market demographics are paramount.

This article is related to the literature on matching and bargaining, although it differs in two key respects. ${ }^{1}$ First, instead of a pairwise matching process, a setting in which offers are publicly observed and opened to acceptance by anyone in the market is considered. While strict bilateral negotiations may characterize the institutional and legal characteristics present in some trading environments, a model with open public offers seems more appropriate in many situations, housing and financial markets, for example.

Second, this article considers stochastic arrival of traders instead of the deterministic entry processes common in other work. With random arrivals the trajectory of the market is not determined completely by its initial mix of buyers and sellers. Instead, every finite level of excess demand is reached with positive probability. For example, a situation in which spot conditions favor buyers even though long-run demographics favor sellers can arise

1. The pioneering articles on matching and bargaining are Rubinstein and Wolinsky [1985, 1990], Gale [1986, 1987], Wolinsky [1987], and Binmore and Herrero [1988]. 
quite naturally. Analyzing stochastic entry also yields some novel insights such as the fact that long-side agents prefer higher market volatility to lower or the fact that the option value from waiting to trade is always positive.

This article is also related to the studies by Vincent [1992] and Merlo and Wilson [1993a, 1933b]. Vincent considers a class of deterministic infinite horizon games in which a seller of an indivisible object faces two or more identical buyers. He shows that it may be possible to support implicitly collusive subgame perfect equilibria in which the buyers capture all the surplus. Vincent argues that sellers may use sealed bids rather than public offers in order to thwart implicit collusion. Although implicitly collusive equilibria can exist in the setting of Section III below, attention throughout this article is restricted to pure-strategy-Markovperfect (stationary) equilibria in which implicit collusion is not possible. Merlo and Wilson [1993a, 1993b] also analyze stochastic $\mathrm{N}$-person infinite horizon bargaining games. Unlike the setting of this article, however, they consider a fixed set of players, and allow nature to determine the size of the pie to be divided among them from period to period.

The basic model and key result are presented in the next section. Also, it is shown that impatience continues to influence the equilibrium division of surplus even when the time between rounds of play shrinks to zero. Markets in which only sellers post prices are considered in Section III. Sellers receive some additional surplus when they possess the exclusive power to post prices, although the magnitude of the incremental surplus they obtain depends on several factors. An intuitive dynamic adjustment process which guarantees that any level of excess demand will eventually be neutralized is considered in Section IV. Under this adjustment process the expected duration of a buyers' or sellers' market is easily calculated, and it is possible to compute the steady-state distribution of excess demand and equilibrium prices. A brief conclusion is presented in Section $\mathrm{V}$, and all of the proofs and two technical lemmas appear in the Appendix.

\section{THe MODEL}

Suppose that there are an infinite number of potential buyers each wishing to purchase one unit of an indivisible homogeneous good and an infinite number of potential sellers each possessing one unit of the good for sale. The respective valuations of buyers 
and sellers are normalized to one and zero. Buyers and sellers are all risk neutral, discount between periods at interest rate $R>0$, and have reservation payoffs of zero. ${ }^{2}$ The horizon is infinite, and all aspects of the environment are common knowledge.

Let $b \in\{0,1,2, \ldots\}$ and $s \in\{0,1,2, \ldots\}$ denote, respectively, the number of buyers and sellers "in the market" at some point in time. For simplicity, imagine that at most one additional buyer and one additional seller can enter the market from period to period. ${ }^{3}$ The probability that a new buyer and no new seller enters is $p \in$ $(0,1)$, and the probability that a new seller and no new buyer enters is $q \in(0,1) .{ }^{4}$ All arrivals are statistically independent, and they occur at the beginning of each period.

Following the arrival stage, all agents on the short side of the market post offers. If $b=s$, so that neither side is short, then the offering side is selected randomly with probability $1 / 2 .{ }^{5}$ An offer by agent $i$ specifies a price $\pi_{i} \in[0,1]$ at which she is willing to trade. Offers in a given period are posted simultaneously and publicly. Each agent $j$ on the nonoffering side of the market responds to one proposal of his choice, $\rho_{j} \in$ ACCEPT $\pi_{i}$, REJECT $\left.\pi_{i}\right\} .{ }^{6}$ If an offer receives at least one acceptance, then it is executed, and the trading parties exit the market and never return. If an offer receives more than one acceptance, then trade is consummated with one of the acceptors chosen at random with equal probability. Agents not party to a trade remain in the market.

It is useful to define three types of spot markets. A buyers' market occurs when excess demand is negative, $b<s$; a sellers' market occurs when excess demand is positive, $b>s$; and a

2. Most of the algebra is cleaner with the interest rate $R$ rather than the discount factor, $\delta \equiv(1+R)^{-1}$.

3 . An alternative arrival process yielding the same results is to suppose that $k$ buyers and sellers deterministically enter the market each period, and that a single "noise buyer" enters with probability $p$ and a single "noise seller" enters with probability $q$. Since all the results are independent of $k$, it is normalized to zero.

4. If $\beta$ and $\sigma$ denote the respective arrival probabilities of a buyer and a seller, then independence implies that $p \equiv \beta(1-\sigma)$ and $q \equiv \sigma(1-\beta)$.

5 . The "coin tossing" assumption when $b=s$ is made purely for symmetry. An asymmetric market in which sellers always post prices is considered in Section III. The assumption here that the short side of the market posts offers can be replaced with any mechanism in which any $m$ agents post offers to which the remaining $b+$ $s-m$ agents respond, provided that there are enough offers and responses for the short side to clear, $\min \{b, s\} \leq m \leq \max \{b, s\}$.

6. Only pure strategy profiles in which offering agents post nonrandom prices to which agents on the other side respond nonrandomly are considered. This ignores the potential for equilibrium coordination failure which can occur if agents use mixed strategies. For instance, several respondents might randomly decide to tender acceptances to the same offer, leaving some equally good or better offers without an acceptance. Equilibrium coordination failure is an interesting and important topic, but is beyond the scope of this study. 
balanced market occurs when excess demand is nil, $b=s$. Obviously, when the market is unbalanced, some agents on the long side will be unable to trade, and will have to wait for the arrival of future partners. There are also three types of long-run markets corresponding to the demographic situations $p<q, p>q$, and $p=q$.

Propositon 1. Consider the trading game, with $b$ buyers and $s$ sellers that begins immediately following the arrival stage. The unique expected payoffs to buyers and sellers in any pure-strategy-Markov-perfect equilibrium of this game are given, respectively, by

$$
U(b-s) \equiv \begin{cases}{\left[\left(\frac{1}{2}\right)+w(p, q, R)\right] \alpha^{b-s}(p, q, R)} & \text { if } b \geq s \\ 1-V(b-s) & \text { if } b \leq s\end{cases}
$$

and

$$
V(b-s) \equiv \begin{cases}{\left[\left(\frac{1}{2}\right)+w(q, p, R)\right] \alpha^{s-b}(q, p, R)} & \text { if } s \geq b \\ 1-U(b-s) & \text { if } s \leq b\end{cases}
$$

where

$$
w(p, q, R) \equiv \frac{q-p}{2 \sqrt{(p+q+R)^{2}-4 p q}},
$$

and

$$
\alpha(p, q, R) \equiv \frac{1}{2 p}\left[p+q+R-\sqrt{(p+q+R)^{2}-4 p q}\right] .
$$

In equilibrium the terms of trade depend both on long-run demographics and spot-market conditions. To see this, first consider a balanced market. The extent to which a trader's expected equilibrium payoff differs from the symmetric split of $1 / 2$ depends on the value of $w(p, q, R)=-w(q, p, R)$. This is positive if and only if $q>p$. So, when sellers are more apt to arrive, demographics favor buyers, and the bargaining position of sellers is weakened relative to the 50-50 split. On the other hand, suppose that demographics are neutral, $p=q$. Then, $w(p, q, R)=0$, and only the degree of imbalance in the spot market influences the equilibrium terms of trade.

The function $\alpha(p, q, R)<1$ is an endogenously determined discount factor that depends directly on equilibrium strategies and, 
therefore, indirectly on the parameters of the model. Specifically, $\alpha^{b-s}(p, q, R)$ and its counterpart $\alpha^{s-b}(q, p, R)$ specify the respective disadvantages to buyers and sellers of being on the long side of the market. In equilibrium the short side of the market clears in each period, and the terms of trade are set so as to leave all agents on the long side just indifferent about trading in the current period or waiting for arrival of a future partner. From period to period, excess demand increases by one with probability $p$, decreases by one with probability $q$, and remains the same with probability $1-$ $p-q$. In other words, excess demand follows a type of random walk process that drifts up if $p>q$ and drifts down if $p<q$. The probability that a sellers' market with excess demand of $b-s$ will eventually become balanced is simply

$$
\alpha^{b-s}(p, q, 0)= \begin{cases}1 & \text { if } p \leq q \\ (q / p)^{b-s} & \text { if } p \geq q .\end{cases}
$$

If the process governing excess demand does not drift up, then this probability is one. However, if the process drifts up, then the probability that a sellers' market will eventually be neutralized decreases with the size of the current imbalance. Likewise, $\alpha^{s-b}(q, p, 0)$ gives the probability of eventually neutralizing a buyers' market. The expected equilibrium payoff to buyers in a sellers' market, $[(1 / 2)+w(p, q, R)] \alpha^{b-s}(p, q, R)$, is the expected present value of waiting to buy in a balanced market, and the expected equilibrium payoff to sellers in a buyers' market, $[(1 / 2)+w(q, p, r)]$ $\alpha^{s-b}(q, p, R)$, is the expected present value of waiting to sell in a balanced market. Hence, the equilibrium is Walrasian since longside agents are always indifferent between trading in the spot market and waiting to trade.

Notice that the equilibrium terms of trade depend on the absolute rather than on the relative numbers of buyers and sellers in the market. For instance, the equilibrium price is the same if the market is composed of one seller and two buyers or 101 sellers and 102 buyers. This is because the terms of trade are determined by the marginal trader, and the presence of an extra 100 inframarginal buyers and sellers makes no difference. The equilibrium price is determined by the magnitude of excess, not total, demand and supply. This, depends critically upon the lack of any coordination failures; i.e., all short-side agents who post the equilibrium price receive at least one acceptance. If acceptors chose among identical offers randomly as in a mixed strategy equilibrium, the expected number of short-side agents not receiving an acceptance would 
depend on the total number of buyers and sellers on hand due to equilibrium coordination failure (see footnote 6). In this case, the equilibrium price in a market with one seller and two buyers would be higher than in a market with 101 sellers and 102 buyers.

For finite numbers of buyers and sellers, a positive interest rate, and arrival rates strictly between zero and one, the equilibrium price given in Proposition 1 is strictly greater than zero and strictly less than one. ${ }^{7}$ This is because there is always a nonzero chance of eventually returning to balance. If demographics are not neutral, however, the market drifts stochastically over time toward a situation in which the side with the smaller arrival rate receives all the surplus. The rate of convergence depends on $R$ as well as on the difference between $p$ and $q$. For instance, in the limit as $R$ tends to zero, convergence is instantaneous, which yields

$$
U(b-s)=1-V(b-s)= \begin{cases}1 & \text { if } p<q \\ \frac{1}{2} & \text { if } p=q \\ 0 & \text { if } p>q\end{cases}
$$

When there is no impatience, the type of agent with the smaller arrival rate can costlessly bide his time until the market is arbitrarily far from balance, obtaining a payoff arbitrarily close to one. The equilibrium terms of trade in the spot market reflect this situation by immediately conferring all the surplus to the type of agent with the smaller arrival rate. By contrast, as $R$ tends to infinity, the market approaches a one-shot trading game in which the short side gets all of the surplus regardless of arrival rates: ${ }^{8}$

$$
U(b-s)=1-V(b-s)= \begin{cases}1 & \text { if } b<s \\ \frac{1}{2} & \text { if } b=s \\ 0 & \text { if } b>s\end{cases}
$$

Thus, when agents are patient, market demographics are paramount in establishing the terms of trade; and when they are

7. In unbalanced markets the equilibrium price is just the sellers' expected payoff $V(b-s)$. When the market is balanced, however, $V(0)$ is the expected payoff to sellers before it is known whether they or the buyers will be selected to make offers. If sellers are selected, then the equilibrium price is $1-(\delta / 2)+w(q, p, R)$; and if buyers are selected, it is $(\delta / 2)+w(q, p, R)$. Note that as the time between offers goes to zero, the equilibrium price when $b=s$ converges to $V(0)=(1 / 2)+w(q, p, r)$ regardless of which side makes offers, where $r$ is the continuous time interest rate.

8. Vincent [1992] investigates nonstationary equilibria of a class of infinite horizon trading games. He finds that it may be possible to support equilibria in which long-side agents (remarkably) garner all the surplus, by threatening to start a price war in the event of defection. 
impatient, the degree of imbalance in the spot market becomes the critical factor.

An important related issue concerns the period length. In most market settings, the real time between bargaining rounds is negligible, and should, therefore, have negligible economic impact. For this reason, it is important to consider the expected equilibrium payoffs to buyers and sellers in the limit game as the period length is squeezed to zero. Fortunately, this is easily done.

Begin by partitioning each original time period into $N$ subperiods of length $1 / N$. In order to keep the expected number of arrivals of buyers and sellers constant over a given interval of real time, suppose that the probability a buyer (and no seller) arrives in a subperiod is $p / N$ and the probability a seller (and no buyer) arrives in a subperiod is $q / N$. Further, suppose that arrivals in any two subperiods are independent and that $N$ is "large enough" that the probability of multiple arrivals in a subperiod is negligible. ${ }^{9}$ Similarly, in order to hold constant the degree of discounting over a given interval of real time, define the interest rate between subperiods by

$$
R_{N} \equiv e^{r / N}-1
$$

where $r>0$ is the continuous time interest rate. Finally, suppose that the market opens in each subperiod with the same trading rules as before.

Observe that

$$
w\left(\frac{p}{N}, \frac{q}{N}, R_{N}\right)=w\left(p, q, N R_{N}\right) \text { and } \alpha\left(\frac{p}{N}, \frac{q}{N}, R_{N}\right)=\alpha\left(p, q, N R_{N}\right) .
$$

To compute the equilibrium payoffs as the real time $1 / N$ between rounds of play goes to zero, recall that $\lim _{N \rightarrow \infty} N R_{N}=r$. So, the expected equilibrium payoffs in the continuous time limit of the game are also given by (1) and (2), except that $R$ is replaced by $r$. The continuous time limit of the market game is more natural than the discrete time formulation. So, the continuous time limit is analyzed below, although the main difference is only that $r$ supplants $R$ in the notation.

Note that even though the real time between rounds of play is negligible in the continuous time limit, impatience still plays an important role in determining the equilibrium division of surplus.

9. In fact, since joint arrival of a buyer and seller results in the same expected equilibrium payoffs as no arrivals, the probability of a joint arrival $\left(\beta \sigma / N^{2}\right.$ in the notation of note 4) can safely be ignored. 
This is because agents on the long side of the market who are "frozen out" of a trade must wait for arrival of a partner, and the amount of time they must wait will amost surely be nonnegligible. This is clearest in a setting of bilateral bargaining, $b=s=1$. In this case, the trader whose type has the higher arrival rate is more anxious to close a deal, because any delay in consummating a trade is more likely to result in the arrival of a trader who competes with him rather than one who competes with his counterpart. So, when $b=s=1$, the respective equilibrium payoffs to the buyer and seller in the continuous time limit are $1 / 2+w(p, q, r)$ and $1 / 2-w(p, q, r)$ even though the time between bargaining rounds is nil.

Another important issue concerns the equilibrium division of surplus and market volatility. Suppose that $p=q \equiv \lambda$. Then, in the continuous time limit buyers and sellers both enter the market according to independent Poisson processes with arrival rates of $\lambda$, and $\lambda$ is both the expected number of trades and the variance of arrivals per period. Expressions (1) and (2) can be used to calculate the expected equilibrium payoff to long-side agents in this setting:

$$
Y(b-s)=\frac{1}{2}\left[1+\frac{r}{2 \lambda}-\sqrt{\left(1+\frac{r}{2 \lambda}\right)^{2}-1}\right]^{|b-s|} .
$$

It is straightforward to check that this expression is increasing in $\lambda$. The question is whether this is due to an increase in expected arrivals or an increase in variance. In fact, (3) is unchanged if arrivals are augmented by a deterministic flow of buyers and sellers, so $Y_{\lambda}>0$ stems from a change in the variance of arrivals. In other words, long-side agents prefer higher market volatility. Another point evident from (3) is that a decrease in $\lambda$ is equivalent to an increase in $r$. Thus, as the arrival rate of new traders approaches zero, the equilibrium payoffs converge to the one-shot outcome in which the short side gets all the surplus.

Before proceeding to the next section, a few words are in order regarding the sensitivity of Proposition 1 to behavior off the equilibrium path. Expressions (1) and (2) are quite robust with respect to deviations from equilibrium strategies. The reason for this is that the terms of trade depend only on excess demand, and this is not affected by deviations. For instance, if a seller posts a price higher than the equilibrium level buyers will prefer to wait rather than accept her offer. On the other hand, if she posts a price lower than the equilibrium level, she will certaintly get an acceptance, and may succeed in luring several hopeful acceptors away 
from other sellers. In either case, however, excess demand at the end of the period will be $b-s$, and as long as strategies are Markovean (memoryless), the equilibrium price in ensuing periods will be unaffected. ${ }^{10}$

\section{ONE-SIDED OFFERS}

Markets with symmetric price-posting rules are important to study because they isolate the impact on the equilibrium terms of trade due solely to market conditions and demographics, and they suppress the influence of pricing conventions. Of course, markets with symmetric price-posting rules are rare. More commonly, one side of the market or the other always posts prices to which the other side responds. For instance, buyers typically post prices in labor markets, and sellers post prices in most retail markets. The following result pertains to markets in which sellers post prices ("ask" markets), but the analogous result holds in markets where buyers make offers ("bid" markets).

Proposition 2. The unique expected payoffs to buyers and sellers in any pure-strategy-Markov-perfect equilibrium of the market game when sellers always post prices are given, respectively, by

$$
\tilde{U}(b-s) \equiv \begin{cases}{\left[\left(\frac{1}{2}\right)+w(p, q, r)-x(p, q, r)\right] \alpha^{b-s}(p, q, r)} & \text { if } b \geq s \\ 1-\tilde{V}(b-s) & \text { if } b \leq s,\end{cases}
$$

and

$$
\tilde{V}(b-s) \equiv \begin{cases}{\left[\left(\frac{1}{2}\right)+w(q, p, r)+x(p, q, r)\right] \alpha^{s-b}(q, p, r)} & \text { if } s \geq b \\ 1-\tilde{U}(b-s) & \text { if } s \leq b\end{cases}
$$

where

$$
x(p, q, r) \equiv \frac{r}{2 \sqrt{(p+q+r)^{2}-4 p q}} .
$$

The terms of trade in unbalanced markets are determined entirely by the indifference of long-side agents to trade at the equilibrium price. So, the only time that the power to post prices matters directly is when the market is balanced, in which case the

10. If strategies are not Markovean, nonstationary equilibria as in Vincent [1992] may exist. 
sellers receive extra surplus of $x(p, q, r)$ relative to their expected payoff under the symmetric pricing rule. Of course, since equilibrium payoffs in unbalanced markets are anchored by the balanced market payoffs, the power to post prices has indirect influence on the terms of trade under all market conditions.

The range of $x(p, q, r)$ is [0,1/2], and it is increasing in the rate of time preference, $r$, with $x(p, q, 0)=0$ and $\lim _{r \rightarrow \infty} x(p, q, r)=1 / 2$. When agents are perfectly patient, payoffs are determined completely by demographics, and the power to post prices confers no benefits to sellers. As impatience grows, however, the power to post prices becomes increasingly meaningful. In the limit as impatience becomes infinite, so that the situation is analogous to a one-shot game, the power to post prices ensures that sellers receive all the surplus in a balanced market. (In an unbalanced market, all the surplus goes to the short side in a one-shot game regardless of the pricing protocol.) The reason that the power to post prices becomes more important as agents become less patient is clear. Under the symmetric pricing protocol, the agents not chosen to make offers in a balanced market will get a chance to make counterproposals before any real time elapses. When sellers possess the exclusive ability to post prices, however, the only alternative open to buyers is to wait for the market to drift out of balance. The more unbalanced the market becomes, the less important is the pricing protocol. In equilibrium, of course, buyers do not wait for the market to drift out of balance because the terms of trade in the spot market incorporate the value of this option. As the degree of impatience rises, the option value of waiting falls, and the ability to post prices takes on greater significance. To summarize, the power to post prices is more important (1) the closer the market is to balance, (2) the more heavily agents discount, and (3) the lower the arrival rates.

\section{LONG-RUN EQUILIBRIUM}

The model is extended in this section by introducing a simple dynamic adjustment process which ensures that the market always returns to balance. Without such a process it was shown above that excess demand drifts over time toward positive or negative infinity depending on relative arrival rates. Moreover, even when demographics are neutral, there is no impetus for the market to move toward balance, and the expected duration of a spell of imbalance is infinite. 
In the short run the equilibrium price given in Propositions 1 and 2 is calibrated so as to clear the short side of the market and to leave agents on the long side just indifferent about trading in the current period. This is what is known as a "stock" equilibrium (see Gale [1987]). In many settings, however, it seems likely that a long-run or "flow" equilibrium will also obtain. That is, arrival rates of buyers and sellers will respond to imbalances in the spot market so as to equate long-run supply and demand. Instead of drifting off to positive or negative infinity, expected excess demand will be zero in the long run. The simplest equilibrating process of this kind is as follows. Redefine $q$ to be the arrival rate of agents on the short side of the market and $p$ to be the arrival rate of long-side agents, and assume that $p<q$. When the market is balanced, suppose that both buyers and sellers arrive at rate $p$. Finally, return to the symmetric pricing protocol explored in Section II. ${ }^{11}$

Proposition 3. Consider the market game with the dynamic adjustment process just described. The unique expected payoff to long-side agents in any pure-strategy-Markov-perfect equilibrium of this game is given by

$$
Y(b-s)=\frac{1}{2} \alpha^{|b-s|}(p, q, r), \quad|b-s|=0,1,2, \ldots
$$

The expected equilibrium payoff to short-side agents is 1 $Y(b-s)$.

In balanced markets, buyers and sellers face symmetric situations under the assumed arrival process. ${ }^{12}$ Hence, the expected equilibrium terms of trade are the 50-50 split. Of course, the market will eventually "step" out of balance when a new trader arrives. The payoff to the long side after this occurs is just the symmetric payoff $1 / 2$ discounted by $\alpha(p, q, r)$, which is just the expected present value of waiting to trade until the market becomes balanced again. The more unbalanced the market becomes, the longer the expected waiting time, and the more heavily the balanced market payoff $1 / 2$ is discounted.

Under the assumed dynamic adjustment process, the market has impetus to move toward balance, and any level of positive or negative excess demand will ultimately be neutralized. In fact, calculating the expected duration of a buyers' or sellers' market

11. It is straightforward to account for markets with one-sided offers by simply adding and subtracting $x(p, q, r)$ to the balanced market payoffs.

12. It is possible to incorporate asymmetric rates of adjustment for positive and negative levels of excess demand, but the solution is algebraically formidable. 
under the assumed adjustment process is a simple matter. Suppose that there is a queue of $|b-s|>0$ long-side agents waiting to trade. Since the net expected arrival of short-side agents is $q-p$ per period, the expected time before the market becomes balanced is just

$$
T(b-s)=\frac{|b-s|}{q-p} .
$$

Notice that as the difference between $q$ and $p$ approaches zero, the expected duration of the imbalance goes to infinity regardless of its magnitude. This is because a market with neutral demographics is just as likely to take a step away from balance as toward it. That is, it behaves like a symmetric random walk.

Under the dynamic adjustment process, excess demand does not tend to positive or negative infinity in the long run. Instead, it settles down into a steady-state distribution.

Proposition 4. The steady-state distribution of excess demand $f(b-s)$ under the assumed adjustment process is given by

$$
f(b-s)=\left(\frac{1-(p / q)}{1+(p / q)}\right)\left(\frac{p}{q}\right)^{|b-s|} .
$$

Intuitively, (4) gives the probability that excess demand will be $b-s$ in the long run. More specifically, given any initial level of excess demand $b_{0}-s_{0}$, calculate the probability that it will be $b-s$ after an interval of time $T$ has elapsed. Then, the long-run or steady-state distribution is obtained by taking the limit as $T$ tends to infinity of this probability. In the limit, of course, the probability of being in state $b-s$ is independent of the initial state $b_{0}-s_{0}$.

The mass function given in (4) is a symmetric distribution centered on zero. In the long run the market is expected to have no excess demand. Observe that as $p / q$ approaches 1 , probability mass is shifted to the tails of the distribution because the net impetus for the market to move toward balance is dampened. Similarly, as $p / q$ goes to zero, mass is transferred to the center. Indeed, when $p / q=$ 0 , all of the probability mass is concentrated at $b-s=0$, and the market deterministically settles at balance with no traders and no arrivals in the long run. Another way of seeing this is to calculate the variance of the distribution,

$$
\operatorname{var}(b-s)=E\left[(b-s)^{2}\right]=\frac{2(p / q)}{[1-(p / q)]^{2}},
$$


as $p / q$ approaches 1 , this becomes infinite becuase the distribution is spread flat, and as $p / q$ approaches zero, the variance goes to zero because the distribution becomes concentrated at its center.

Finally, since Proposition 3 specifies the unique equilibrium price associated with any level of excess demand, and Propostion 4 gives the long-run (steady-state) distribution of excess demand, it is straightforward to combine these results to obtain the steadystate distribution of equilibrium prices.

\section{CONCLUSION}

The simple model analyzed in this article helps provide intuition about the determinants of bargaining power and price formation in dynamic markets. Specifically, when agents on the long side of the market possess the option to wait for the arrival of future trading partners, the terms of trade in the spot market must honor the value of this option. In fact, the option to wait exists in balanced markets as well, which skews bargaining power toward the side of the market with the smaller arrival rate even when the real time between bargaining rounds is negligible.

There are several directions in which this model could be extended. Consideration of heterogeneous agents and private information on one or both sides of the market as in Samuelson [1992] or Fudenberg, Levine, and Tirole [1987] would lend additional realism to the setting studied here. Also, investigating the duration and efficiency of trading relationships in "rental" markets as in Deere [1988] seems germane. Extensions like these are intriguing and potentially important because they may yield additional insights regarding who winds up holding the short end of the stick, and just how short it might be.

\section{APPENDIX}

The following two Lemmas are used to ease the proof of Proposition 1.

LEMMA 1. In any pure-strategy-Markov-perfect equilibrium, all agents on the same side of the market receive the same expected payoff.

Proof of Lemma 1. Suppose to the contrary that $U$ is the lowest expected equilibrium payoff received by a buyer and $\bar{U}$ is the highest expected equilibrium payoff received by a buyer in a market 
with excess demand of $b-s$, where $\underline{U}<\bar{U}$. Note, however, that a buyer receiving $\underline{U}$ can obtain a payoff arbitrarily close to $\bar{U}$ by undercutting, that is, by posting and accepting arbitrarily higher prices than a buyer who otherwise receives $\bar{U}$. But, this implies that no equilibrium payoff $\underline{U}<\bar{U}$ exists. The argument is identical for sellers.

QED

LEMma 2. The short side of the market clears each period in any pure-strategy-Markov-perfect equilibrium.

Proof of Lemma 2. Suppose without loss of generality that $b>$ $s$, so that sellers post prices. Denote the expected equilibrium payoff of sellers by $V(n)$ and the expected equilibrium payoff of buyers by $U(n)$, where $n \equiv b-s$. If a seller posts a price $\pi$ that no buyer accepts in equilibrium, then neither he nor the buyers must expect to do worse by waiting a period. That is,

$$
\pi \leq \delta[p V(n+1)+q V(n-1)+(1-p-q) V(n)],
$$

and

$$
1-\pi \leq \delta[p U(n+1)+q U(n-1)+(1-p-q) U(n)] .
$$

Adding these and collecting terms gives

$$
\begin{aligned}
1 \leq \delta\{p[U(n+1)+V(n+1)] & +q[U(n-1)+V(n-1)] \\
+ & (1-p-q)[U(n)+V(n)]\} .
\end{aligned}
$$

Note, however, that $U(m)+V(m) \leq 1$ so that (A.1) implies that $1 \leq \delta$, a contradiction.

QED

Proof of Proposition 1. Let $n \equiv b-s$, and fix a pure-strategyMarkov-perfect equilibrium. Use Lemmas 1 and 2 to write the expected payoffs to buyers and sellers in this equilibrium, respectively, as $U(n)$ and $V(n)$. Suppose first that $b-s \geq 1$ so that buyers form the long side of the market. By Lemma 1 the buyers who consummate trades in the current round get the same expected payoff as the buyers who do not, or

$$
\begin{aligned}
U(n)=\delta[p U(n+1)+q U(n-1)+(1-p-q) U(n)], & \\
n & =1,2, \ldots .
\end{aligned}
$$

Similarly, if $b-s \leq-1$, the sellers who consummate trades in the 
current round get the same expected equilibrium payoff as the sellers who do not, or

$$
\begin{aligned}
V(n)=\delta[p V(n+1)+q V(n-1)+(1-p-q) V(n)], \\
n=-1,-2, \ldots
\end{aligned}
$$

These can be rewritten as second-order homogeneous difference equations with general solutions,

$$
U(n)=c_{1} \alpha_{1}^{n}+c_{2} \alpha_{2}^{n}, \quad n=1,2, \ldots
$$

and

$$
V(n)=d_{1} \beta_{1}^{-n}+d_{2} \beta_{2}^{-n}, \quad n=-1,-2, \ldots,
$$

where

$$
\alpha_{j}=\frac{1}{2 p}\left[p+q+R+(-1)^{j} \sqrt{(p+q+R)^{2}-4 p q}\right], \quad j=1,2,
$$

and $\beta_{j}=(p / q) \alpha_{j}$. It is straightforward to check that these roots are real and distinct, and that they satisfy $\alpha_{2}>1>\alpha_{1}>0$ and $\beta_{2}>$ $1>\beta_{1}>0$. This implies that the constants $c_{2}=d_{2}=0$ because the solutions are otherwise unbounded, which would contradict the fact that agents' payoffs cannot be greater than one. Next, let $n=$ 1 , and substitute the solution into (A.2) to get $c_{1}=U(0)$. Similarly, let $n=-1$, and substitute into (A.3) to find $d_{1}=V(0)$.'Using this and Lemma 2 yields

$$
U(n)=1-V(n)=U(0) \alpha^{n}, \quad n=1,2, \ldots
$$

and

$$
V(n)=1-U(n)=V(0) \beta-n,
$$$$
n=-1,-2, \ldots,
$$

where $\alpha \equiv \alpha(p, q, R)$ and $\beta \equiv \alpha(q, p, R)$. The next step is to find the balanced market equilibrium payoffs. Indeed, if multiple equilibrium payoffs exist, the above analysis indicates that they must stem from multile equilibria in balanced markets. Let $\bar{u}$ and $u$ be, respectively, the highest (supremum) and lowest (infimum) balanced market equilibrium payoffs to buyers when they are selected to make offers. Similarly, let $\bar{v}$ and $v$ be, respectively, the highest and lowest balanced market equilibrium payoffs to sellers when they are selected to make offers. Also, define $\bar{U}(0)$ and $\underline{U}(0)$, respectively, to be the highest and lowest equilibrium expected payoffs to buyers in a balanced market before the offering side has 
been determined, and define $\bar{V}(0)$ and $V(0)$ to be the analogous expected payoffs to sellers. By Lemma 2 , a balanced market clears in equilibrium, so

$$
\begin{aligned}
& \bar{U}(0)=0.5 \bar{u}+0.5(1-\underline{v}), \\
& \underline{U}(0)=0.5 \underline{u}+0.5(1-\bar{v}), \\
& \bar{V}(0)=0.5 \bar{v}+0.5(1-\underline{u}), \\
& \underline{V}(0)=0.5 \underline{v}+0.5(1-\bar{u}) .
\end{aligned}
$$

Next, in equilibrium, buyers can offer sellers no less than the worst they can expect to get by waiting a period, or

$$
\text { (A.7) } \bar{u} \leq 1-\delta\{p[1-\alpha \bar{U}(0)]+q \beta \underline{V}(0)+(1-p-q) \underline{V}(0)\} \text {. }
$$

On the other hand, buyers need offer sellers no more than the best they can expect to get next period:

$$
\text { (A.8) } \underline{u} \geq 1-\delta\{p[1-\alpha \underline{U}(0)]+q \beta \bar{V}(0)+(1-p-q) \bar{V}(0)\} \text {. }
$$

The same arguments apply when sellers make offers, which yields

$$
\text { (A.9) } \bar{v} \leq 1-\delta\{p \alpha \underline{U}(0)+q[1-\beta \bar{V}(0)]+(1-p-q) \underline{U}(0)\},
$$

and

$$
\text { (A.10) } \underline{v} \geq 1-\delta\{p \alpha \bar{U}(0)+q[1-\beta \underline{V}(0)]+(1-p-q) \bar{U}(0)\} \text {. }
$$

Adding these four inequalities and substituting from (A.6) gives

$$
(\bar{u}-\underline{u})+(\bar{v}-\underline{v}) \leq[1-\delta \sqrt{(p+q+R)-4 p q]}[(\bar{u}-\underline{u})+(\bar{v}-\underline{v})] .
$$

However, this inequality implies that $\bar{u}=u$ and $\bar{v}=v$. That is, the pure-strategy-Markov-perfect expected equilibrium payoffs are unique. Using this, (A.7) and (A.8) reduce to

(A.11) $u=1-\delta\{p[1-\alpha U(0)]+q \beta V(0)+(1-p-q) V(0)\}$,

and (A.9) and (A.10) reduce to

(A.12) $v=1-\delta\{p \alpha U(0)+q[1-\beta V(0)]+(1-p-q) U(0)\}$.

Also, (A.6) becomes

$$
\begin{aligned}
& U(0)=0.5 u+0.5(1-v), \\
& V(0)=0.5 v+0.5(1-u) .
\end{aligned}
$$


Solving the system (A.11), (A.12), and (A.13) gives

$$
U(0)=\frac{1}{2}+w(p, q, R),
$$

and

$$
V(0)=\frac{1}{2}+w(p, q, R) .
$$

Finally, combining these expressions with (A.4) and (A.5) establishes the claim.

QED

Proof of Proposition 2. The proof is similar to that for Proposition 1. The main difference is that because sellers post prices even in balanced markets, (A.11) and (A.12) become, respectively,

$$
\tilde{U}(0)=1-\tilde{V}(0)
$$

and

$$
\tilde{V}(0)=1-\delta\{p \alpha \tilde{U}(0)+q[1-\beta \tilde{V}(0)]+(1-p-q) \tilde{U}(0)\} .
$$

Solving these establishes the claim.

QED

Proof of Proposition 3. Use the same method as in the proofs of Propositions 1 and 2.

QED

Proof of Proposition 4. Let $n \equiv(|b-s|)$, and begin by considering $n>0$. Over the long run, probability mass $f(n-1)$ is moved "up" to $n$ at rate $p$; probability mass $f(n+1)$ is moved "down" to $n$ at rate $q$; and probability mass $f(n)$ "stays" at $n$ at rate $1-p-q$. Thus, if $f(n)$ is to be stationary, it must satisfy

$$
f(n)=q f(n+1)+(1-p-q) f(n)+p f(n-1) .
$$

This can be recast as a second-order homogeneous difference equation with general solution,

$$
f(n)=c_{1}\left(\frac{p}{q}\right)^{n}+c_{2}
$$

Since the $f(n)$ 's must sum to one as $n$ runs from zero to infinity, it must be that $c_{2}=0$. To find $c_{1}$, consider $n=0$. Over the long run, probability mass $f(1)$ is moved down to zero at rate $2 q$, and 
probability mass $f(0)$ stays at zero at rate $1-2 p$. This yields

$$
f(0)=2 q f(1)+(1-2 p) f(0),
$$

or

$$
f(0)=(q / p) f(1)=c_{1} .
$$

Finally, since the probability mass must sum to one,

$$
c_{1}\left[1+2 \sum_{n=1}^{\infty}\left(\frac{p}{q}\right)^{n}\right]=1,
$$

which implies that

$$
c_{1}=\frac{1-(p / q)}{1+(p / q)} .
$$

TEXAS A\&M UNIVERSITY

\section{REFERENCES}

Binmore, Kenneth, and Marie Herrero, "Matching and Bargaining in Dynamic Markets," Review of Economic Studies, LV (1988), 17-34.

Deere, Donald, "Bilateral Trading as an Efficient Auction over Time," Journal of Political Economy, XCVI (1988), 100-15.

Fudenberg, Drew, David Levine, and Jean Tirole, "Incomplete Information Bargaining with Outside Opportunities," Quarterly Journal of Economics, CII (1987), $37-50$.

Gale, Douglas, "Bargaining and Competition Part 1: Characterization; Part 2: Existence," Econometrica, LIV (1986), 785-806.

, "Limit Theorems for Markets with Sequential Bargaining," Journal of Economic Theory, XL (1987), 20-54.

Merlo, Antonio, and Charles Wilson, "A Stochastic Model of Sequential Bargaining with Complete Information and Transferable Utility," C. V. Starr Center for Applied Economics, New York University, RR93-05, 1993a.

Merlo, Antonio, and Charles Wilson, "A Stochastic Model of Sequential Bargaining with Complete Information and Nontransferable Utility," C. V. Starr Center for Applied Economics, New York University, RR93-06, 1993b.

Rubinstein, Ariel, and Asher Wolinsky, "Equilibrium in A Market with Sequential Bargaining," Econometrica, LIII (1985), 1133-50.

Rubinstein, Ariel, and Asher Wolinsky, "Decentralized Trading, Strategic Behavior, and the Walrasian Outcome," Review of Economic Studies, LVII (1990), 63-78.

Samuelson, Larry, "Disagreement in Markets with Matching and Bargaining," Review of Economic Studies, LIX (1992), 177-85.

Vincent, Daniel, "Modeling Competitive Behavior," Rand, XXIII (1992), 590-99.

Wolinsky, Asher, "Matching, Search, and Bargaining," Journal of Economic Theory, XLII (1987), 311-33. 